\title{
The Effects of Nonmagnetic Bolus on Contralateral Breast Skin Dose during Tangential Breast Irradiation Therapy
}

\author{
Young-Jin Won ${ }^{1}$, Jae-Hwan Cho ${ }^{2}$, and Sung-chul Kim ${ }^{3 *}$ \\ ${ }^{1}$ Department of Radiation Oncology, Inje University, Iisan Paik Hospital, Iisan 10380, Korea \\ ${ }^{2}$ Department of Radiological Technology, Ansan College, Ansan 15518, Korea \\ ${ }^{3}$ Department of Radiological Science, Gachon University Medical Campus, Yeonsugu, Incheon 21936, Korea
}

(Received 23 November 2015, Received in final form 2 January 2016, Accepted 5 January 2016)

\begin{abstract}
In this study the contralateral breast skin dose was decreased. It was to apply the results to the clinical study after analysis of different radiation dose amounts to contralateral breast with nonmagnetic bolus and without nonmagnetic bolus. A Rando phantom was computed tomography (CT) simulated, five treatment plans were generated: open tangents, open field in field, wedge 15, wedge 30, and intensity-modulated radiotherapy (IMRT) plan with 50.4 Gy to cover sufficient breast tissue. Contralateral breast skin dose was measured at 8 points using a glass dosimeter. The average contralateral breast dose using nonmagnetic bolus showed better excellence in decreasing the absorbed dose in the order of $168 \pm 11.1 \mathrm{cGy}, 131 \pm 10.2 \mathrm{cGy}(29 \%), 112 \pm 9.7 \mathrm{cGy}$ $(49 \%)$, and $102 \pm 9.5 \mathrm{cGy}(64 \%)$ than changing the treatment plan. This study focused on decreasing the effect of scattered dose by use of a nonmagnetic bolus on the contralateral breast during radiotherapy in breast cancer patients and an intriguingly significant decrease was observed parallel to the opposed beam.
\end{abstract}

Keywords : nonmagnetic bolus, breast dose, tangential breast therapy

\section{Introduction}

Breast cancer is currently the second leading cause of female cancer, followed by thyroid cancer, and according to the National Cancer Information Center, Korea [1], there were 15,942 incidences of breast cancer in the year 2011. Although the treatment involves use of a tangential method as a traditional radiation therapy, recent treatment includes several other methods: field in field planning and inverse planning method by intensity-modulated radiation therapy (IMRT), and volumetric modulated arc therapy (VMAT). These methods adhere to the tangential method in their expanded aspect. However, the advanced treatments bear the inevitable risk of contralateral breast skin leakage dose during treatment. According to John et al. average leakage dose in contralateral breast skin by traditional radiation therapy was $282 \mathrm{cGy}$ and maximum leakage dose of $710 \mathrm{cGy}$ was reported [2]. A study by Starkschall et al. [3] explained that leakage was due to the collimator that controlled the beam shape and the multi-

CThe Korean Magnetics Society. All rights reserved.

*Corresponding author: Tel: +82-32-820-4364

Fax: +82-32-820-4361, e-mail: ksc@gachon.ac.kr leaf collimator. Recently, following development of conventional radiation therapy, Williams et al. [4] reported a significantly diminished leakage dose such as 100 200 cGy in the case of a 50.4 Gy dose. In addition, John et al. warned of a possible increase in incidence of secondary cancer in women 45 years of age or younger or in patients who are overexposed to contralateral breast skin dose [2]. In this study, in order to decrease the contralateral breast skin dose, we used a nonmagnetic bolus that is very accessible during radiation therapy and its density is similar to that of skin. Although higher density materials such as metals are more effective in blocking radiation, they lead to higher treatment error and the patient does not feel comfortable. Although vaseline is very useful for expressing the body line, it is unlikely economical as it takes too much time while wrapping to create enough thickness on the body. The surface of the current nonmagnetic bolus is coated with flexible materials and it possesses properties of gel-formation, therefore it has better contact with skin. In addition, we can confirm the ink-line (easily purchasable) by laser in the treatment room after setting up the patient; we can also use nonmagnetic bolus by cutting with scissors. Because there are few studies on the measurement of radiation dose around 
the center of the contralateral breast, we tried to measure the radiation dose around the entrance dose point. Therefore, the goal of this study was to analyze different radiation dose amounts to the contra-lateral breast with nonmagnetic bolus and without nonmagnetic bolus for application of the results in clinical study.

\section{Methods}

\subsection{Phantom set-up and image acquisition}

We used the Rando phantom (ART-300; The Alderson radiation therapy phantom-300, RSD; Radiology Support Devices NC., USA), which has the shape of the human body and is made of material equivalent to tissue from head to pelvic region. The breast margin was marked using a wire between upper and lower skin margins during radiation treatment of the chest area of the phantom. Eight different points were measured in the phantom and on the other side of the breast, the points at which the treatment was applied were marked on the inside, center, and outside as 2,8 , and $14 \mathrm{~cm}$ away from the medial margin (Fig. 1). Each point has three levels of marking point, high, medium, and low at a distance of $8 \mathrm{~cm}$. However, due to the limitation of the Rando phantom size, we only marked high and low points on the outer side at a distance of $14 \mathrm{~cm}$. Using computer tomography, $5 \mathrm{~mm}$ slice thickness images of the phantom were acquired and these images were transferred to obtain the planning system in order to apply the radiation treatment planning.

\subsection{Treatment plan}

Images of lung, heart, bronchus, and spinal cord were positioned onto each slice image and were re-constructed as $3 \mathrm{D}$ images in order to achieve treatment planning (Fig. 2). These images were applied to 5 different treatments, including open, open field-in-field (FIF), wedge $15^{\circ}$, wedge $30^{\circ}$, intensity modulated radiation therapy (IMRT),

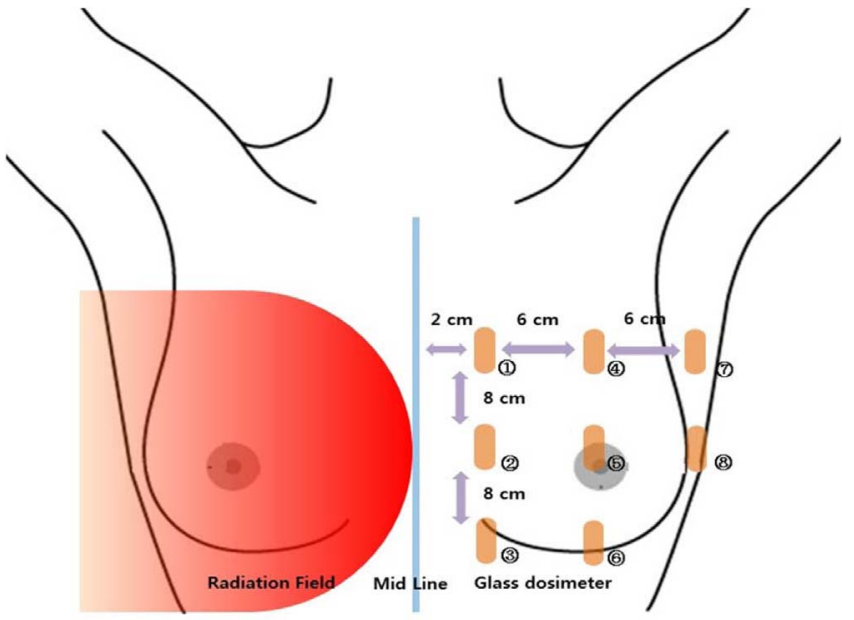

Fig. 1. (Color online) Treating area of radiation therapy and located measuring spots of glass dosimeter on the contralateral breast.

and wedge plan (lateral beam of $234^{\circ}$ ). Based on the previous report by Williams et al. [4] on enhancement of dose using medial wedge, this treatment was excluded from the current study. All treatments were carried out to simulate a general clinical breast treatment plan using the Rando phantom and efforts were made to include the breast tissue of the phantom by providing maximum protection to the lung. In addition, both medial beam and lateral beam were set up to gantry $54^{\circ}, 234^{\circ}$ and the energy level was set to $6 \mathrm{MV}$. In addition, the multi leaf collimator (MLC) was adjusted during the treatment in order to minimize the absorption of dose by lung. The field size was $18 \mathrm{~cm}$ (Y-axis) to cover sufficient breast tissue and prescribed dose used was 50.4 Gy (Fig. 3). The treatment of IMRT was planned as an inverse planning method and had the same direction of the open field treatment plan. The monitor unit (MU) of each plan is shown in Table 1.

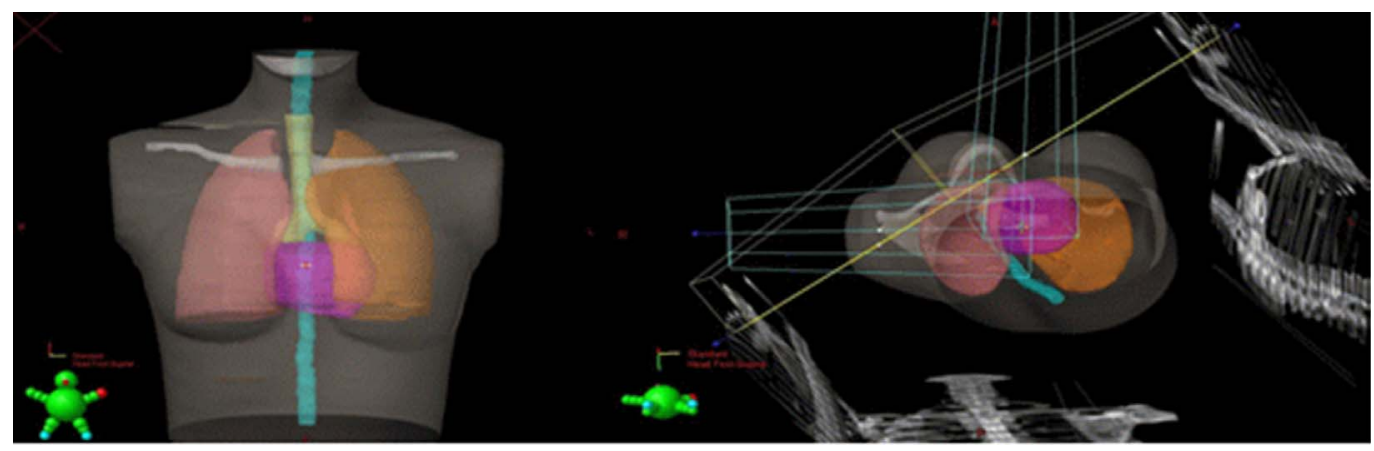

Fig. 2. (Color online) Drawn major organs in CT images and reconstructed the $3 \mathrm{D}$ image for treatment plan. 


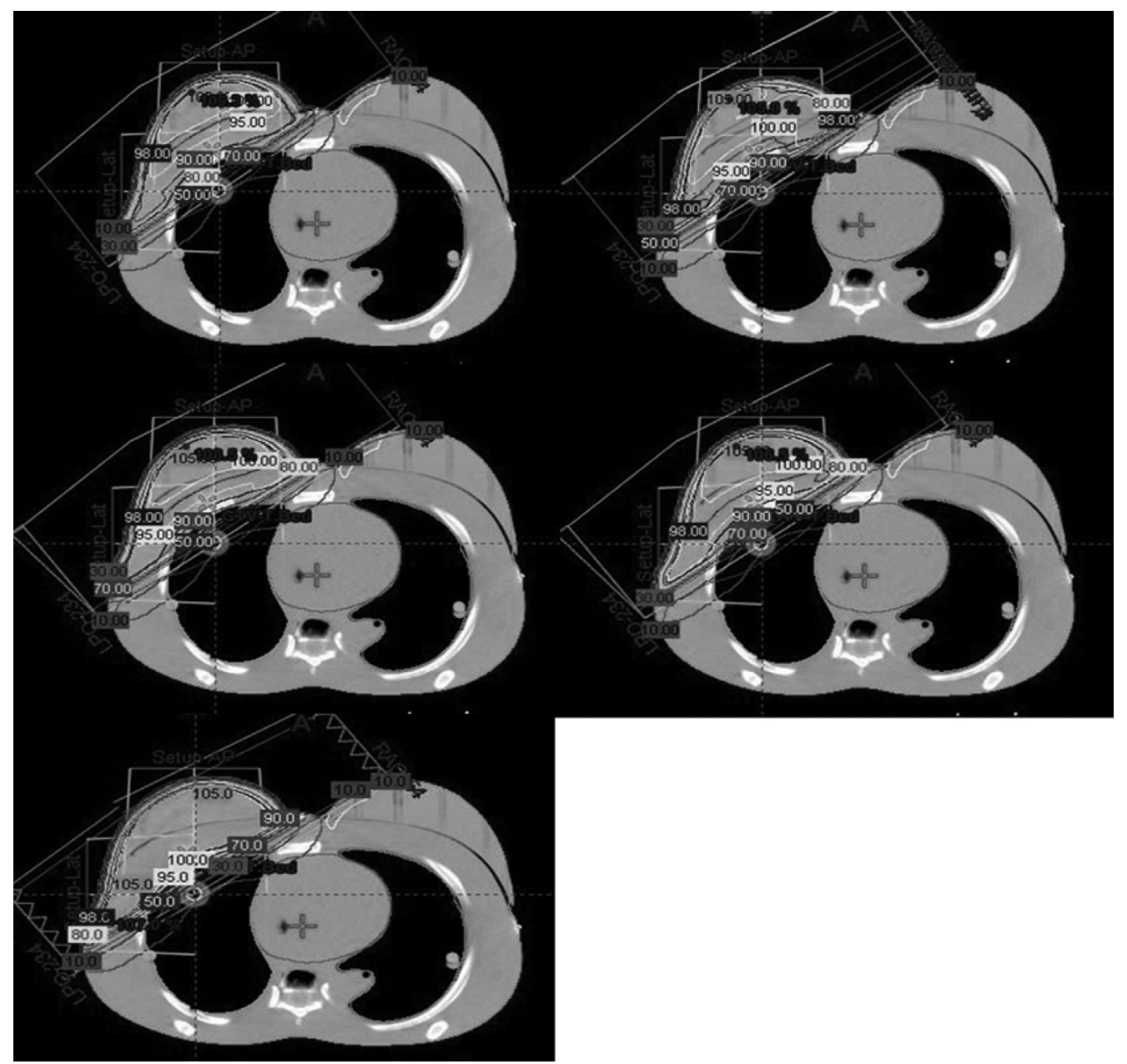

Fig. 3. Treatment plan of Rando phantom (from left upper side open, open-FIF, wedge 15, Wedge 30, IMRT).

Table 1. Calculation result of MU value for each treatment plan.

\begin{tabular}{lccccc}
\hline \hline & Open & Open-FIF & Wedge-15 & Wedge-30 & IMRT \\
\hline LAO & 2,790 & 3,300 & 2,790 & 2,760 & 4,690 \\
RPO & 2,560 & 2,800 & 4,060 & 5,640 & 3,600 \\
Total & 5,370 & 6,100 & 6,850 & 8,400 & 8,290
\end{tabular}

LAO: left anterior oblique, RPO: Right posterior oblique

\subsection{Measurement of glass dosimeter}

\subsubsection{Calibration of dosimeter}

Twenty glass dosimeters (GD-302M, Asahi Technology glass corporation, Japan) were used for calibration. In this research, the converted values were taken without any corrections and they would be presented as (glass dose for dosimeter). Although this does not identify the variations between the glass dosimeters and would increase the uncertainty in the final results, it could be a more practical method. In this study, the calibration factors of 6 glass dosimeters were obtained as the acquisition of the baseline data. Firstly, the net glass dose $\mathrm{G}$ was defined to be

$$
\mathrm{G}_{\mathrm{i}}=\mathrm{G}_{\mathrm{i}, \text { read }}-\mathrm{G}_{\mathrm{i}, \mathrm{pre}}
$$

Where $G_{p r e}$ is the reading from the dosimeter before irradiation and $G_{i, \text { read }}$ is the reading after irradiation. Although the unit of $G$ is supposed to be $\mu G y$ in the reader, the unit of $\mathrm{G}$ would be presented as $\mu \mathrm{Gy}$-gr (glass reading) or $\mathrm{mGy}$-gr in this paper to be distinguished with the unit of absorbed dose. Therefore, the calibration constant of the glass dosimeter is following [5].

$$
\mathrm{N}_{\mathrm{i}}=\frac{\mathrm{D}_{\mathrm{ref}}}{\mathrm{R}}\left[\frac{\mathrm{mGy}}{\mathrm{mGy}}-\mathrm{gr}\right]
$$

To measure the element factor of the glass dosimeter, a specially produced acryl phantom was used to calibrate the glass dosimeter using $200 \mathrm{cGy}$ doses. The dose of the acryl phantom showed different measurements wherever the area was off from the center point because of the off axis ratio. The measurement was set up with the follow- 
Table 2. Organs at risk (OAR) estimate of LINAC in the Acryl phantom. (Shadow is in Fig 5-B)

\begin{tabular}{cccccccccc}
\hline \hline & 1 & 2 & 3 & 4 & 5 & 6 & 7 & 8 & 9 \\
\hline A & 1.041 & 1.044 & 1.042 & 1.041 & 1.041 & 1.041 & 1.042 & 1.044 & 1.041 \\
B & 1.044 & 1.041 & 1.038 & 1.035 & 1.034 & 1.035 & 1.038 & 1.041 & 1.044 \\
C & 1.042 & 1.038 & 1.031 & 1.018 & 1.014 & 1.018 & 1.031 & 1.038 & 1.042 \\
D & 1.043 & 1.037 & 1.029 & 1.013 & 1 & 1.013 & 1.029 & 1.037 & 1.043 \\
E & 1.042 & 1.038 & 1.031 & 1.018 & 1.014 & 1.018 & 1.031 & 1.038 & 1.042 \\
F & 1.044 & 1.041 & 1.038 & 1.035 & 1.034 & 1.035 & 1.038 & 1.041 & 1.044 \\
G & 1.041 & 1.044 & 1.042 & 1.041 & 1.041 & 1.041 & 1.042 & 1.044 & 1.041 \\
\hline
\end{tabular}

Table 3. Result of light emitting rate (LER) reflecting organs at risk (OAR).

\begin{tabular}{cccccccccccc}
\hline \hline No. & 1 & 2 & 3 & 4 & 5 & 6 & 7 & 8 & 9 & 10 \\
\hline LER & 1.041 & 1.064 & 1.062 & 0.982 & 1.060 & 1.059 & 1.067 & 1.106 & 1.047 & 1.083 \\
\hline No. & 11 & 12 & 13 & 14 & 15 & 16 & 17 & 18 & 19 & 20 \\
\hline LER & 1.127 & 1.094 & 1.098 & 1.087 & 1.074 & 1.090 & 1.092 & 1.120 & 1.092 & 1.129 \\
\hline
\end{tabular}

ing conditions: $16 \mathrm{~mm}$ depth $\left(\mathrm{D}_{\max } \mathrm{spot}\right), 6 \mathrm{MV} \mathrm{X}$-ray, $\mathrm{X}$ ray field size $20 \times 20 \mathrm{~cm}^{2}$, dose rate 300 using LINAC with water phantom (Table 2). In this study, each element factor (Table 3 ) of the glass dosimeter was carried out by 200 cGy, marked in black and white and used as postcalibration. In general, the dose of X-ray of the glass dosimeter tends to be averted because UV laser emission is different at every reading [6-8] Thus, there is a need to use an internal control as a reference dose and we used three glass dosimeters (\#1 to 3 ) that pre-measured absolute value of X-ray dose using the water phantom and 200 cGy (condition: $180 \mathrm{MU}, 6 \mathrm{MV}, 300$ Dose rate, field size $10 \times 10 \mathrm{~cm}^{2}, 16 \mathrm{~mm}$ depth). These glass dosimeters were used simultaneously during the reading. Finally, onedirected measurement was carried out due to the angular dependence of X-ray and the measurement was done from the center of the measure point of the glass dosimeter. Tweezers were used to avoid contamination.

\subsubsection{Measurement of dosimeter}

All treatment plans, open field, open FIF, wedge-15, wedge-30, and IMRT were simulated in a similar way as for the glass dosimeter. During the measurement, the glass dosimeter was centered in the cell and the beam was passed through the tangential area of the glass dosimeter due to angular dependence. Subsequently, every treatment plan was measured twice according to $0,3,5$, and $10 \mathrm{~mm}$ of nonmagnetic bolus (Superflab nonmagnetic bolus, Radiation Products Design Inc., USA) (Fig. 4). Due to the limitation of the Rando phantom and subsequent transmission of nonmagnetic bolus near the primary beam, we did not measure the dose decrease at the center point of a $2 \mathrm{~cm}$ inner area.

\subsubsection{Dosimeter analysis}

Pre-annealing of measured dosimeter was carried out at $70^{\circ} \mathrm{C}$ for 30 minutes and the results were read after the temperature had dropped to $30^{\circ} \mathrm{C}$. Sixteen dosimeter results were analyzed (measured twice) and the results from three relative-dosimeters exposed to 200 cGy simultaneously during the data reading were collected. Because the power of the UV laser kept changing during each measurement, the data were analyzed according to relativedosimeter and luminescence rate was also included. Analysis of data from this study was performed using SPSS (IBM SPSS statistics 21, IBM, USA), and the results were rounded up to the hundredth place $(\mathrm{p}<0.05)$.

\section{Results}

Each treatment plan for each point of measurement showed a different X-ray dose, similar to the results from the Rando phantom (Table 4). Compared with the Open Plan, the average measurement of each plan showed the following results: Wedge-15 (277 $\pm 17.3 \mathrm{cGy}, 14.7 \%)$, IMRT $(275 \pm 17.4$ cGy, 13.9\%), Open-FIF $(266 \pm 17.1$ cGy. 11.1\%), Wedge-30 (263 \pm 17.0 cGy, 10.1\%), and Open (236 \pm 15.7 cGy) Plan. Although Wedge-15 showed the highest result and Open plan showed lowest, the difference between them was minimal (41 cGy). Unlike the results shown in Fig. 5, there was no significant effect but overall trend showed dependence of X-ray dose on the increase in MU. For the measurement of different points, the results from the medial-center point at $2 \mathrm{~cm}$ from the center were $803 \pm 4 \mathrm{cGy}, 953 \pm 62 \mathrm{cGy}, 976 \pm$ $30 \mathrm{cGy}, 942 \pm 26 \mathrm{cGy}$, and $986 \pm 16 \mathrm{cGy}$ in respective treatment plans including open, open-FIF, wedge-15, wedge- 

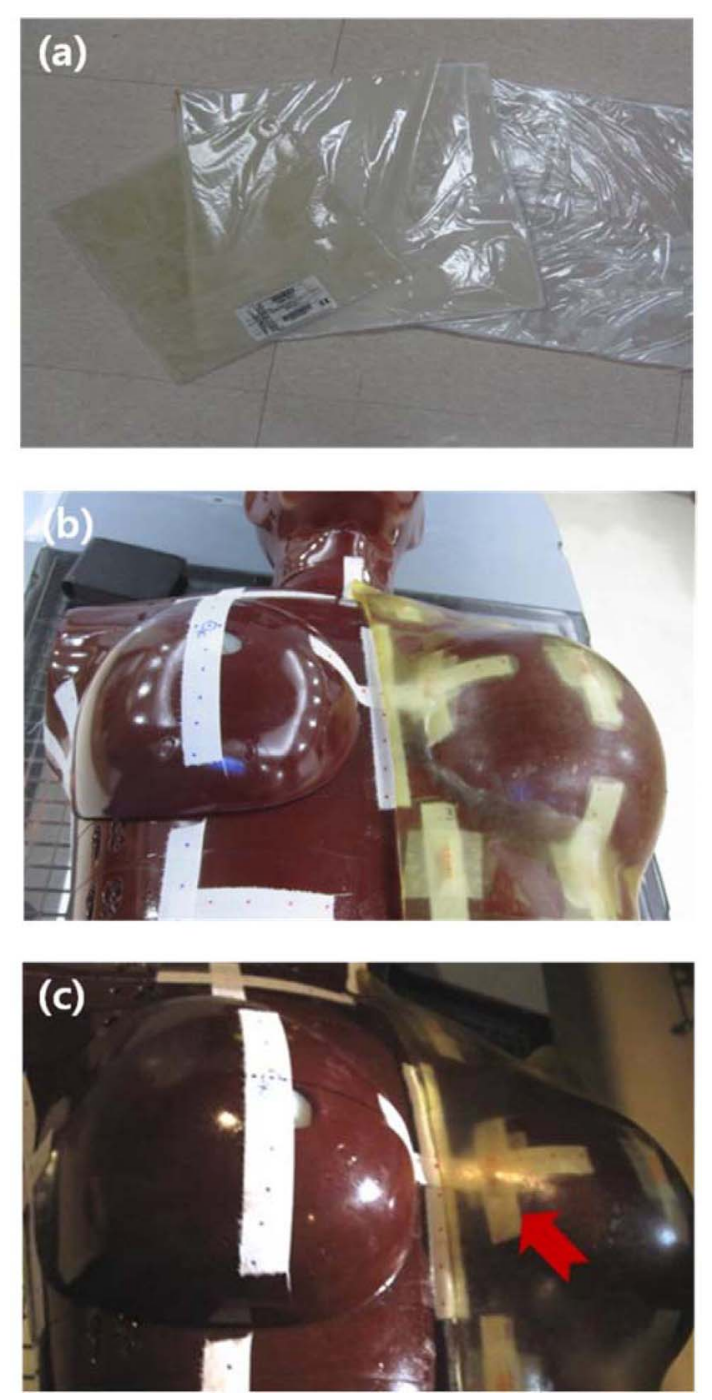

Fig. 4. (Color online) Rando phantom and Nonmagnetic bolus. $\mathrm{A}$ is $3,5,10 \mathrm{~mm}$ Nonmagnetic bolus used in the experiment. $\mathrm{B}$ is Nonmagnetic bolus attached on Rando phantom. $\mathrm{C}$ is The arrow shows trespassing primary radiation onto the $10 \mathrm{~mm}$ Nonmagnetic bolus (Explain that number 2 measuring spot is immeasurable).
30, and IMRT. Thus, we were able to find a significantly high exposure dose. In the center of the breast (sample $\# 5$ ), each treatment plan, including open, open-FIF, wedge15, wedge-30, and IMRT showed $274 \pm 2 \mathrm{cGy}, 293 \pm 7$ cGy, $302 \pm 3$ cGy, $269 \pm 15$ cGy, and $314 \pm 7$ cGy and the results were similar to the average dose (168 cGy) from samples $1 \sim 8$. In addition, the results from the lateralcenter point of the breast (sample \#8) were $54 \pm 1 \mathrm{cGy}$, $57 \pm 2 \mathrm{cGy}, 64 \pm 2 \mathrm{cGy}, 57 \pm 2 \mathrm{cGy}$, and $79 \pm 1 \mathrm{cGy}$ and demonstrated a very low dose. The results from the upper part, samples 1, 4, and 7, and from the down part, samples 3 and 6 were lower than the dose from each sample measured at the center point. The highest dose was the one from the IMRT plan (sample \#2) with the value of $986 \pm 16$ cGy, while the lowest dose, $29 \pm 1$ cGy, was from the Open Plan (sample \#7) measured at the upperlateral-center point. The results from the treatment using nonmagnetic bolus are shown in Table 5. In this measurement, the results from all points except the one from \#2 (Medial-center-point) showed a lower dose than the one without using nonmagnetic bolus. The measurement from \#2 of nonmagnetic bolus showed a significantly higher dose because of its location at the margin of the beam due to the thickness of nonmagnetic bolus (Fig. 4). The

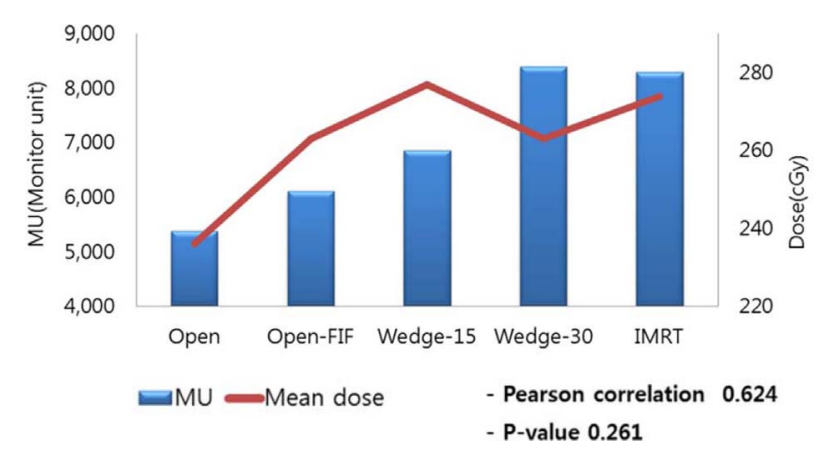

Fig. 5. (Color online) Correlation of MU and absorption dose by treatment plan.

Table 4. Dose each to the treatment plan and increase rate of dose compare open treatment plan with other treatment plan.

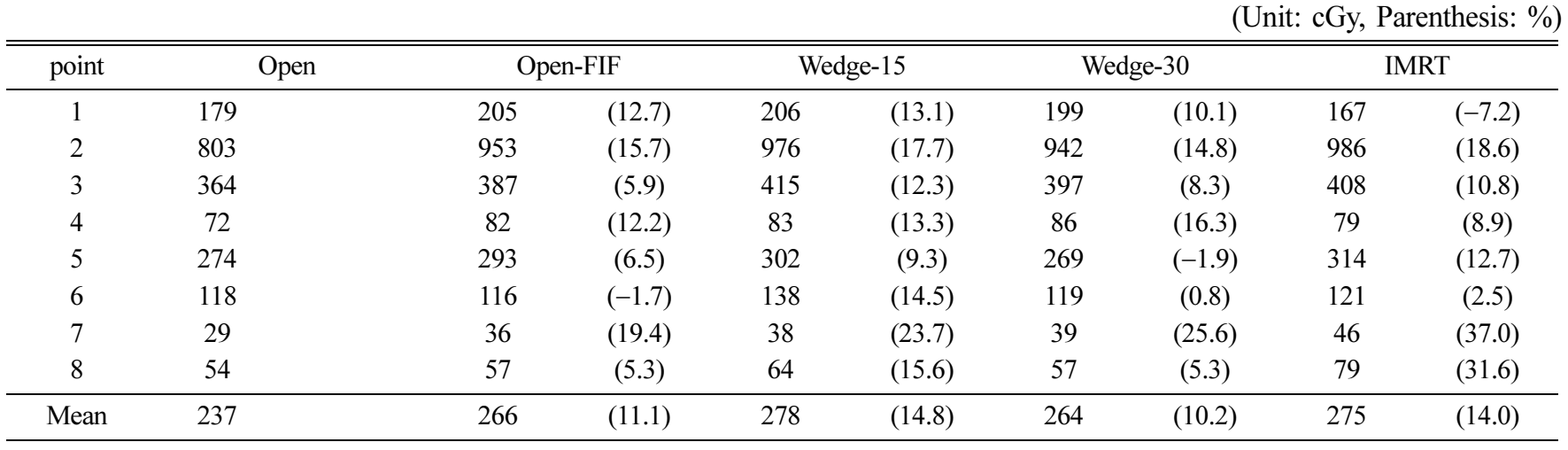


average dose at the open plan except for \#2 showed a decreasing trend from highest to lowest, as shown in
Table 5: $155 \pm 10.9$ cGy for non-nonmagnetic bolus, 124 $\pm 10.3 \mathrm{cGy}(26 \%)$ for $3 \mathrm{~mm}, 108 \pm 9.9 \mathrm{cGy}(44 \%)$ for 5

Table 5. Dose each to the nonmagnetic bolus and decrease rate of dose compare non-nonmagnetic bolus with each nonmagnetic bolus.

(Unit: cGy, Parenthesis: \%)

\begin{tabular}{|c|c|c|c|c|c|}
\hline & & Non-Nonmagnetic bolus & Nonmagnetic bolus $3 \mathrm{~mm}$ & Nonmagnetic bolus $5 \mathrm{~mm}$ & Nonmagnetic bolus $10 \mathrm{~mm}$ \\
\hline \multirow{8}{*}{ Open } & 1 & 179 & $143(-25)$ & $118(-52)$ & $107(-67)$ \\
\hline & 2 & 803 & - & - & - \\
\hline & 3 & 364 & $323(-13)$ & $300(-21)$ & $277(-31)$ \\
\hline & 4 & 72 & $49(-47)$ & $43(-67)$ & $37(-95)$ \\
\hline & 5 & 274 & $214(-28)$ & $182(-51)$ & $133(-106)$ \\
\hline & 6 & 118 & $86(-37)$ & $73(-62)$ & $52(-127)$ \\
\hline & 7 & 29 & $20(-45)$ & $17(-71)$ & $15(-93)$ \\
\hline & 8 & 54 & $33(-64)$ & $26(-108)$ & $23(-135)$ \\
\hline \multicolumn{2}{|c|}{ Mean } & 155 & $124(-26)$ & $108(-44)$ & $92(-69)$ \\
\hline \multirow{8}{*}{ Open FIF } & 1 & 205 & $132(-55)$ & $137(-50)$ & $125(-64)$ \\
\hline & 2 & 953 & - & - & - \\
\hline & 3 & 387 & $296(-31)$ & $266(-45)$ & $255(-52)$ \\
\hline & 4 & 82 & $57(-44)$ & $44(-86)$ & $47(-74)$ \\
\hline & 5 & 293 & $242(-21)$ & $191(-53)$ & $187(-57)$ \\
\hline & 6 & 116 & $95(-22)$ & $64(-81)$ & $62(-87)$ \\
\hline & 7 & 36 & $26(-38)$ & $20(-80)$ & $19(-89)$ \\
\hline & 8 & 57 & $30(-90)$ & $25(-128)$ & $22(-159)$ \\
\hline \multicolumn{2}{|c|}{ Mean } & 168 & $125(-34)$ & $107(-57)$ & $102(-64)$ \\
\hline \multirow{8}{*}{ Wedge -15} & 1 & 206 & $154(-34)$ & $118(-75)$ & $84(-145)$ \\
\hline & 2 & 976 & - & - & - \\
\hline & 3 & 415 & $297(-40)$ & $264(-57)$ & $224(-70)$ \\
\hline & 4 & 83 & $54(-54)$ & $52(-60)$ & $37(-124)$ \\
\hline & 5 & 302 & $235(-29)$ & $166(-82)$ & $194(-56)$ \\
\hline & 6 & 138 & $84(-64)$ & $76(-82)$ & $86(-60)$ \\
\hline & 7 & 38 & $25(-52)$ & $25(-52)$ & $22(-73)$ \\
\hline & 8 & 64 & $31(-106)$ & $32(-100)$ & $37(-73)$ \\
\hline \multicolumn{2}{|c|}{ Mean } & 178 & $125(-42)$ & $105(-70)$ & $101(-77)$ \\
\hline \multirow{8}{*}{ Wedge-30 } & 1 & 199 & $176(-13)$ & $155(-28)$ & $146(-36)$ \\
\hline & 2 & 942 & - & - & - \\
\hline & 3 & 397 & $369(-8)$ & $345(-15)$ & $329(-21)$ \\
\hline & 4 & 86 & $81(-6)$ & $76(-13)$ & $50(-89)$ \\
\hline & 5 & 269 & $237(-14)$ & $212(-27)$ & $195(-72)$ \\
\hline & 6 & 119 & $97(-23)$ & $91(-31)$ & $84(-38)$ \\
\hline & 7 & 39 & $35(-11)$ & $38(-3)$ & $31(-42)$ \\
\hline & 8 & 57 & $43(-33)$ & $42(-36)$ & $37(-54)$ \\
\hline \multicolumn{2}{|c|}{ Mean } & 166 & $148(-12)$ & $137(-22)$ & $124(-34)$ \\
\hline \multirow{8}{*}{ IMRT } & 1 & 167 & $113(-48)$ & $94(-78)$ & $77(-117)$ \\
\hline & 2 & 986 & - & - & - \\
\hline & 3 & 408 & $307(-33)$ & $288(-42)$ & $282(-45)$ \\
\hline & 4 & 79 & $60(-32)$ & $46(-72)$ & $41(-93)$ \\
\hline & 5 & 314 & $257(-22)$ & $190(-65)$ & $129(-143)$ \\
\hline & 6 & 121 & $100(-21)$ & $71(-70)$ & $62(-95)$ \\
\hline & 7 & 46 & $33(-39)$ & $20(-130)$ & $17(-171)$ \\
\hline & 8 & 79 & $50(-58)$ & $38(-108)$ & $34(-132)$ \\
\hline \multicolumn{2}{|c|}{ Mean } & 173 & $131(-32)$ & $106(-63)$ & $91(-89)$ \\
\hline
\end{tabular}


Table 6. Reliability dose of decrease rate in 3,5,10mm Nonmagnetic bolus by multiple comparison analysis (Tukey method).

\begin{tabular}{cccccc}
\hline \hline $\begin{array}{c}\text { Standard } \\
\text { nonmagnetic bolus }\end{array}$ & $\begin{array}{c}\text { Compare nonmagnetic } \\
\text { bolus }\end{array}$ & Mean difference & P-value & 95\% Confidence interval \\
\hline $0 \mathrm{~mm}$ & $3 \mathrm{~mm}$ & 36.68 & 0.156 & -8.47 & 81.81 \\
& $5 \mathrm{~mm}$ & 55.03 & 0.010 & 9.88 & 100.17 \\
& $10 \mathrm{~mm}$ & 65.41 & 0.001 & 20.27 & 110.55 \\
\hline
\end{tabular}

$\mathrm{mm}$, and $92 \pm 9.4 \mathrm{cGy}(69 \%)$ for $10 \mathrm{~mm}$. For the openFIF plan, the results showed a decreasing trend, as follows: $168 \pm 11.1 \mathrm{cGy}, 125 \pm 10.1 \mathrm{cGy}(34 \%), 107 \pm$ $9.6 \mathrm{cGy}(57 \%)$, and $102 \pm 9.4 \mathrm{cGy}(64 \%)$. For the Wedge15 plan, a decrease in values was observed as $178 \pm 11.5$ cGy, $125 \pm 10.2$ cGy (42\%), $105 \pm 9.1$ cGy (70\%), and $101 \pm 9.1$ cGy $(77 \%)$. Wedge-30 plan also a showed similar decreasing trend: $166 \pm 11.2 \mathrm{cGy}, 148 \pm 10.8 \mathrm{cGy}$ $(12 \%), \quad 137 \pm 10.4$ cGy $(22 \%)$, and $124 \pm 10.2 \mathrm{cGy}$ $(34 \%)$. The pattern of results was similar to that of the IMRT plan as per the observed values, $173 \pm 11.4 \mathrm{cGy}$, $131 \pm 10.2 \mathrm{cGy}(32 \%), 106 \pm 9.2 \mathrm{cGy}(63 \%)$, and $91 \pm$ 9.5 cGy $(89 \%)$. Finally, when analyzing the average of each plan, the results showed the following trend: $168 \pm$ 11.1 cGy, $131 \pm 10.2$ cGy (29\%), $112 \pm 9.7$ cGy (49\%), and $102 \pm 9.5 \mathrm{cGy}(64 \%)$ (Table 6$)$. The average dose of non-nonmagnetic bolus showed $\mathrm{p}$ values of 0.156 for 3 $\mathrm{mm}, 0.010$ for $5 \mathrm{~mm}$, and 0.001 for $10 \mathrm{~mm}$, respectively, and there was no significant difference in the results from $3 \mathrm{~mm}$; however, a significant difference in the results from 5 or $10 \mathrm{~mm}$ was observed. In addition, the point at 7 or 8 showed a signiicantly diminished dose, while the point at 1,2 , or 3 showed a minimally decreased dose.

\section{Discussion}

Starkschall et al. [3] explained constant existence of some exposed radiation at contralateral breast dose by each treatment because of leakage from a collimator or instrument head area or the patient's body. So far, many studies on minimizing this side effect have been conducted [9-13], but they have been unsuccessful due to a limited treatment plan and higher MU. Therefore, in this study, we investigated several trials to minimize the radiation dose to the contralateral breast. In the current study, nonmagnetic bolus and Rando phantom model having tissue equivalent materials were employed and applied to several treatment plans including open plan, open-FIF, Wedge- $15^{\circ}$, Wedge- $30^{\circ}$, and IMRT for measurement of absorbed dose at 8 points. The results of measurement by the one without using nonmagnetic bolus were similar to those of other studies. According to a study by Jone et al. [2], the measured contralateral breast dose was 282 cGy.
Chougule A reported measurement of 155 255 cGy dose near the nipple. In a study by Ajay et al. [8], IMRT plan showed 362 cGy for the contralateral breast dose at a distance of $4 \mathrm{~cm}$ from the other side of treatment and the one from wedge- $30^{\circ}$ was $565 \mathrm{cGy}$ at the same point. A study by Williams et al. [4] also reported the dose from upper or low part of the outer area as $120 \mathrm{cGy}$, and both lateral $330 \mathrm{cGy}$, respectively (in the presence of IMRT treatment). In this study, the average breast dose from individual treatment plans was $268 \mathrm{cGy}$ and the average dose at a distance $2 \mathrm{~cm}$ apart from upper and lower part was 287 cGy for IMRT, 310.5 cGy for Wedge- $15^{\circ}$, and 298 cGy for Wedge- $30^{\circ}$. The average breast dose was similar to the results reported by Jone et al. [2] and other measurement points also showed similar results in line with the reported study. The dose near the treatment site was found to be higher than the data reported by Ajay et al. [14]. However, this could be due to the possible measurement at the inner point of measurement. The results obtained using nonmagnetic bolus showed further decrease in value with increasing thickness of nonmagnetic bolus in every treatment plan. In particular, the Wedge plan with the highest dose showed higher skin dose because of scattered dose from collimator and wedge filter. A tendency of $30 \%$ decrease in dose was observed as compared with the open plan when only $3 \mathrm{~mm}$ of nonmagnetic bolus was used. Therefore, treatment using nonmagnetic bolus is proposed to be extremely effective when compared with the current treatment plan as the current treatment possesses some risk of exposure. Jone et al reported a $40 \%$ decrease in dose when patients aged younger than 45 years old received a contralateral breast dose of $2.82 \mathrm{~Gy}$, and with a relative side effect of 2.61 [2]. In addition, when they received a contralateral breast dose of $1.11 \mathrm{~Gy}$, the relative side effect was 1.54 and showed a $40 \%$ decrease as compared with the previous dose $(2.82 \mathrm{~Gy})$. These results are consistent with the results obtained from the current study. After obtaining results of average breast dose of $264 \mathrm{cGy}$ in the case of the treatment plan without using nonmagnetic bolus, we could predict an average breast dose of $186 \mathrm{cGy}, 129$ cGy, and $92 \mathrm{cGy}$ at $3 \mathrm{~mm}, 5 \mathrm{~mm}$, and $10 \mathrm{~mm}$ of nonmagnetic bolus, respectively, upon calculation of the aver- 
age decrease rate (the average decrease rate was calculated as 29,49 , and $65 \%$, as we could not get the average breast dose due to the measurement point of \#2). If these doses were applied with the results from the study by Jone et al, there would be a $40 \%$ decrease in second cancer occurrence in patients younger than 45 years in the case of $5 \mathrm{~mm}$ of nonmagnetic bolus and a more than $40 \%$ decrease in cancer occurrence in the case of $10 \mathrm{~mm}$ of nonmagnetic bolus. Although the risk of cancer occurrence after breast removal surgery is only less than $3 \%$, as per National Cancer Center, Korea, it is still higher because occurrence of breast cancer per year is 15,000 [1]. We also encountered certain problems in this study during the measurement period, such as the dose at medial-center-point which is the closest to the medial beam margin was increased. There are possible reasons for the observed behavior. The first reason includes the enhancement of scattered dose due to the location of nonmagnetic bolus at beam margin. The second reason is the difference in thickness of breast between the patient and Rand phantom. Patient breast was soft enough to collapse while there was no change in the thickness of the phantom breast. This property deviates the distance from the point to the beam. For that reason, we could not measure the longer distance of $5 \mathrm{~mm}$ of nonmagnetic bolus due to the additional scattered line. In addition, there are several other factors to be considered while using nonmagnetic bolus, such as feeling of inconvenience in patients due to the weight of nonmagnetic bolus and patient's movement after set up. These factors will increase the error, thus detailed attention must be paid during clinical application.

\section{Conclusion}

This study focused on decreasing the effect of the scattered dose by using a nonmagnetic bolus on the contralateral breast during radiation therapy in breast cancer patients and an intriguingly significant decrease was observed for parallel opposed beams. Hence, it is proposed that these results will be of benefit to not only general radiation therapy for breast cancer, but also to the complicated treatment that requires gantry angle after breast removal surgery or to patients, who could be at risk of more exposure of dose from the scattered line.

\section{Acknowledgements}

Young-Jin Won and Jae-Hwan Cho equally contributed to this work. They are co-first authors.

\section{References}

[1] Z. S. Kim, S. Y. Min, C. S. Yoon, H. J. Lee, J. S. Lee, H. J. Youn, H. K. Park, D. Y. Noh, and M. H. Hur, J. Breast Cancer. 17, 99 (2014).

[2] D. B. John, B. H. Elizabeth, B. Maria, S. Marilyn, and B. A. Flannery, N. Engl. J. Med. 326, 781 (1992).

[3] G. Starkschall, F. J. George, and D. L. Zellmer, Med. Phys. 10, 906 (1983).

[4] T. M. Williams, J. M. Moran, S. H. Hsu, R. Marsh, B. Yanke, B. A. Fraass, and L. J. Pierce, Int. J. Radiation Oncology Biol. Phys. 82, 2079 (2012).

[5] Y. M. Moon, D. J. Rhee, J. K. Kim, Y. R. Kang, M. W. Lee, H. J. Lim, and D. H. Jeong, Korean J. Med. Phys. 24, 140 (2013).

[6] J. E. Lah, D. O. Shin, J. Y. Kim, H. S. Hong, C. I. Lim, H. G. Jeong, and T. S. Suh, J. Korea Asso. Radiat. Prot. 31, 181 (2006).

[7] Y. C. Lo, G. Yasuda, T. J. Fitzgerald, and A. U. Marcia, Int. J. Radiat. Oncol. Biol. Phys. 46, 187 (2000).

[8] R. Ramani, S. Russell, and P. O'Brien, Int. J. Radiation Oncology Biol. Phys. 43, 245 (1999).

[9] W. A. Beckham, C. C. Popescu, V. V. Patenaude, E. S. Wai, and I. A. Olivotto, Int. J. Radiation Oncology Biol. Phys. 69, 918 (2007).

[10] C. Arun, J. Can. Res. Ther. 3, 8 (2007).

[11] M. T. Kim, H. K. Lee, Y. C. Heo, and J. H. Cho, J. Magn. 19, 15 (2014).

[12] J. H. Kim, M. S. Han, S. J. Yoo, K. J. Kim, and J. H. Cho, J. Magn. 20, 120 (2015).

[13] J. H. Kim, and J. P. Hong, J. Magn. 20, 155 (2015).

[14] A. K. Bhatnagar, E. Brandner, D. Sonnik, A. Wu, S. Kalnicki, M. Deutsch, and D. E. Heron Breast Cancer Res. Treat. 96, 41 (2006). 